\title{
ABORDAGEM PROCESSUAL EM PESQUISA QUALITATIVA
}

\author{
Kadígia Faccin \& ${ }^{1}$ Bibiana Volkmer Martins \\ Universidade do Vale do Rio dos Sinos - UNISINOS, Rio Grande do Sul, (Brasil)
}

DETALHES DO ARTIGO
Artigo convidado:
Recebido em: 06 de agosto de 2021
Aceito: 26 de novembro de 2021
Disponível online: 10 de dezembro de
2021
Sistema de revisão "Double blind review"
Editor Científico
Ilan Avrichir
Palavras-chaves:
Abordagem Processual
Processo
Pesquisa Qualitativa

\begin{abstract}
RESUMO
O uso da abordagem processual quase triplicou nos últimos sete anos nos estudos organizacionais ${ }^{2}$, principalmente, pelo seu potencial em explicar fenômenos em movimento, dinâmicos, ou seja, processuais em pesquisas qualitativas. Nesse artigo, buscamos apresentar de forma didática os princípios do processo para propiciar aos pesquisadores iniciantes, e mesmo àqueles que já têm alguma experiência com a abordagem, as diferentes possibilidades, recomendações para a condução de uma pesquisa com rigor e exemplos de artigos que utilizaram a abordagem processual. A motivação para a escrita desse artigo veio da constatação, em nossa atividade de orientação de dissertações e teses na área de Estratégias Interorganizacionais, de que a abordagem tem grande potencial de explicar fenômenos complexos de forma dinâmica, analisando a história de como um fenômeno se tornou o que é. E que, por falta de algumas orientações simples, muitos pesquisadores não aproveitam o potencial da abordagem, ou mesmo diminuem as chances de publicações de seus estudos por não a empregarem com rigor metodológico.
\end{abstract}

\section{POR QUE A ABORDAGEM PROCESSUAL?}

A abordagem processual em pesquisa qualitativa permite que se enxergue a realidade organizacional para além da relação causal entre variáveis dependentes e independentes, avaliando a dinâmica das organizações e dos contextos em que se inserem. O foco de um estudo processual é o exame dos padrões temporais, observando como eles se desdobram ou mudam ao longo do tempo (BIZZI; LANGLEY, 2012). A investigação de um determinado processo é útil para analisar a história de como um fenômeno se tornou o que é, e não apenas analisar o que é.

Pela análise processual é possível entender, por exemplo, o processo de criação de um unicórnio, desde seu nascimento até o dia da consagrada ascensão no mercado financeiro. Pode-se, por exemplo, informar se este processo é dividido por algumas fases e qual o padrão de comportamento de cada uma destas fases. É possível ainda, identificar explicar quais os padrões de comportamento que levam a startup de uma fase a outra. Ou seja, explicar "como" e "por que" as mudanças acontecem ao longo do tempo (MOHR, 1982).

E é justamente aí que reside uma grande oportunidade para alcançar novos insights em estudos organizacionais - na análise de processos. Mas, de que são feitos os processos?

\section{A abordagem processual é útil para analisar a história de como um fenômeno se tornou o que é!}

\footnotetext{
${ }^{1}$ Contato do autor E-mail: bibivolkmer@hotmail.com

2 Em busca realizada na Web of Science, no dia 10/12/21, pelas palavras-chave "process approach"; "process data"; "Langley"; "Abdallah"; e "Tsoukas", utilizando o filtro de tipo "artigos" e de área "business" e "management", encontrouse um total de 639 artigos entre os anos de 1969 e 2021. Destes, 60\% de 2015 em diante.
} 


\section{O que é um processo?}

Um processo pode ser entendido como uma ação contínua e regulada que expressa continuidade no desenvolvimento de uma determinada atividade.
Mohr (1982) enfatizou que um processo é uma sequência de eventos que levam a um resultado. Em um processo, é preciso fazer A e B para obter, em seguida, C. Em um desenho muito simples, o autor demonstrou o que é um processo.

Figura 1

\section{Teoria de Processo}

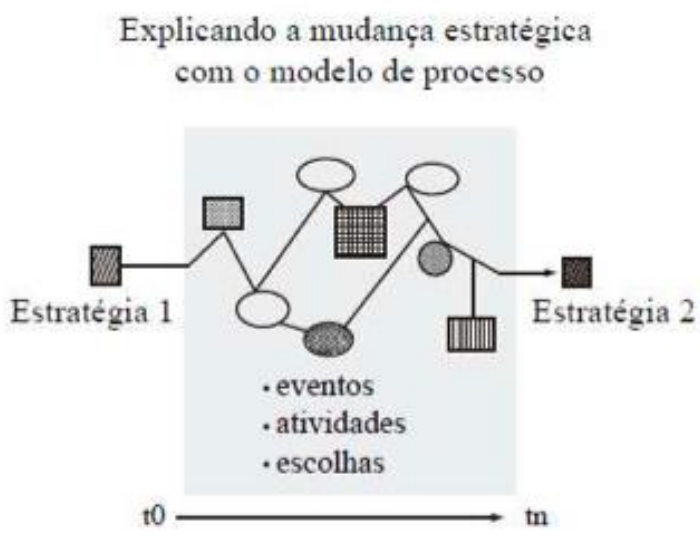

Fonte: Adaptado de Mohr (1982, p. 456)

Assim, pode-se dizer que na análise de um processo se busca entender as atividades, as escolhas e como as escolhas anteriores se relacionam para permitir ao fenômeno ser o que é. Van de Ven (1992;
1995) se refere ao processo como uma sequência de eventos ao longo do tempo, que explicaria como e por que uma entidade organizacional muda, se desenvolve e termina (LANGLEY et al., 2013).

\section{Um processo é um conjunto de eventos relacionados que acontecem ao longo do tempo.}

Se os processos são uma sequência de eventos, como encontrar os eventos? Os eventos são as histórias sobre o que aconteceu e quem fez o quê e quando; ou seja, atividades e escolhas ordenadas ao longo do tempo. Portanto, a abordagem processual qualitativa consiste em organizar uma sequência de eventos.

Eventos são histórias de quem fez o quê, quando e por que...repletas de detalhes!

Uma sequência de eventos é uma entidade conceitual com a qual os pesquisadores estão menos familiarizados. Os eventos geralmente envolvem muitos níveis e unidades de análise que não têm limites bem definidos, e cujo horizonte de tempo costuma variar em termos de precisão, duração e relevância. Eventos são, portanto, aquelas pequenas histórias contadas por alguém que viveu uma situação. Essas pequenas histórias são repletas de detalhes, atores e diversos elementos que podem estar influenciando aquele momento particular. Portanto, dizemos que um evento é uma unidade muito complexa de ser estudada. Para facilitar o estudo dos eventos, vamos fornecer algumas recomendações, baseadas na nossa experiência profissional. 


\section{QUAIS AS PRINCIPAIS RECOMENDAÇÕES PARA INICIANTES EM ABORDAGENS PROCESSUAIS?}

Um processo pode ser estudado de forma retrospectiva ou no momento em que está ocorrendo. Porém, em termos de orientação temporal, iniciantes devem preferir as formas retrospectivas, já que isso facilita fotografar o antes da mudança, no tempo zero (to) e depois da mudança, tempo $n$ (tn). O processo é o que acontece entre estes dois períodos. A fotografia garante que o pesquisador terá clareza sobre o processo que será analisado e qual será a sua unidade de análise. Sempre questione se você é capaz de caracterizar os fenômenos antes e depois da mudança ocorrer.

Ao usar a abordagem processual, você pode optar por variadas técnicas de análise de dados, no texto de
Langley (1999) são enumerados oito diferentes. Sugerimos que você adote duas delas de forma concomitante: a narrativa e o mapa conceitual.

A narrativa consiste na contação da história, a recomposição dos eventos em forma de texto. Já os mapas permitem a apresentação deles de forma visual. Sugerimos o mapa abaixo, de Langley e Truax (1994), para todos nossos alunos que adotam a abordagem processual. Na Figura 2, reproduzimos um mapa como exemplo. Ele explica como uma pequena empresa adota uma determinada tecnologia avaliando as mudanças em cada setor, ao longo do tempo. Em termos teóricos, o artigo já foi superado, mas ainda é um bom exemplo para quem está começando e precisa se inspirar.

\section{Figura 2}

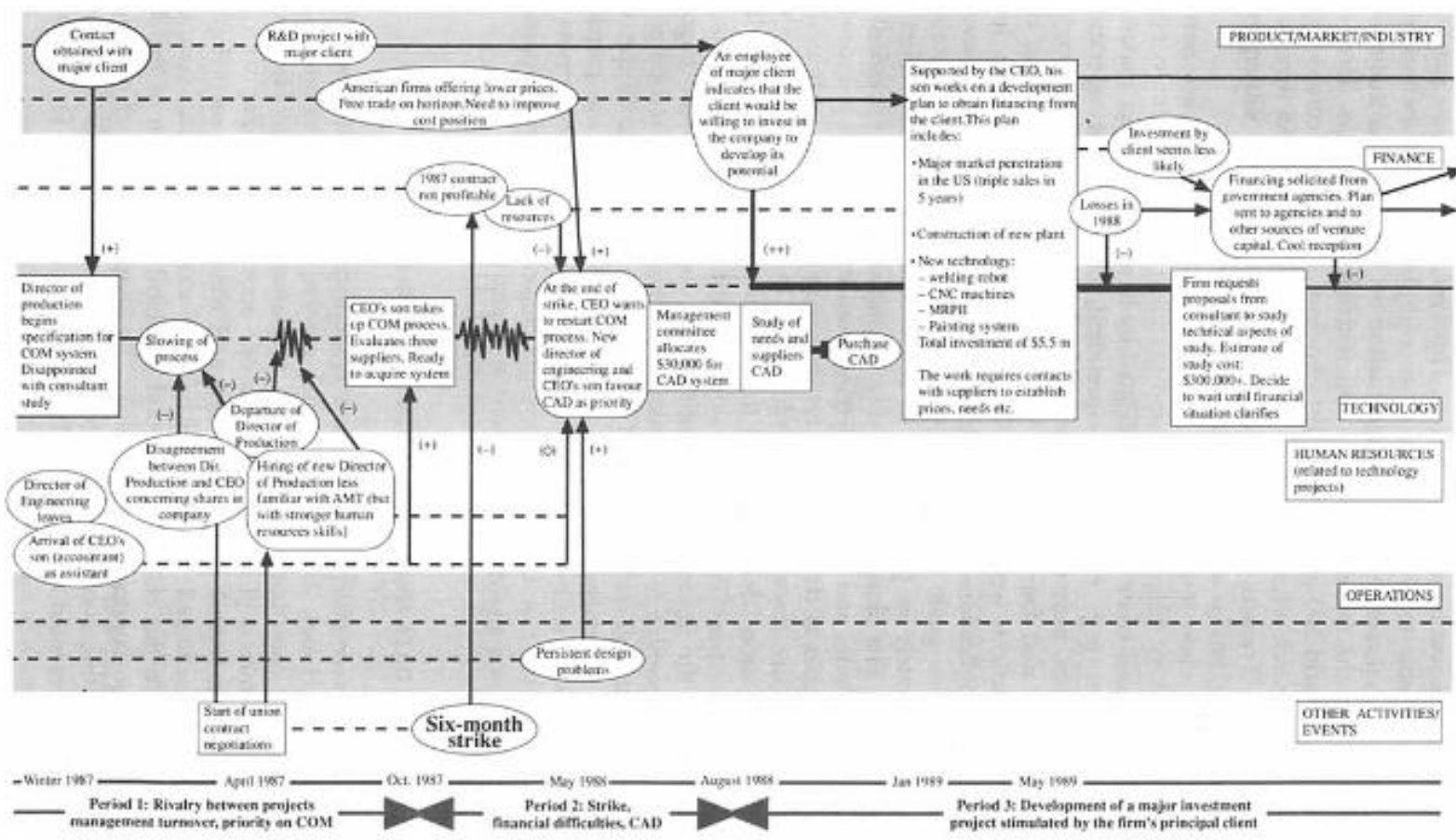

Fonte: Langley e Truax (1994)

Note que o mapa está dividido em fases: períodos 1,2 e 3. As informações para o agrupamento dos eventos em fases surgem naturalmente, da fala dos sujeitos de pesquisa, ou seja, da coleta de dados. Em geral, os pesquisados, de forma unânime, atribuem a determinado "evento" uma mudança de comportamento, ação ou estratégia. No entanto, a sensibilidade do pesquisador para extrair as informações e, posteriormente, organizá-las cronologicamente, são fundamentais. Note que, nem sempre as fases têm a mesma duração, podendo existir fases de 3 meses, 2 dias e outras de 10 anos. Observe que no exemplo, a duração não é constante.

Perceba que existem "raias" ou "piscininhas"como nossos alunos batizaram finanças, tecnologia, operações, mercado. Cada uma destas piscinas que estão destacadas em branco e cinza, contêm os eventos relacionados com este setor na organização e apresentam os links com os acontecimentos anteriores ou subsequentes. Os links são explicados detalhadamente na narrativa. Por isso, sugerimos que o mapa seja acompanhado pela 
narrativa e, mais que isso sugerimos, que o mapa nasça antes da narrativa. Isso ajuda a construir um storytelling (LANGLEY, 1999) que faça sentido e que evidenciei as ligações dos eventos ao longo do tempo. As piscinas podem ser descobertas no campo (de forma mais indutiva) ou podem ser inspiradas em algum quadro conceitual já existente.
É importante enfatizar ainda, que o estudo processual se baseia na busca de padrões, de repetibilidade de como as coisas acontecem dentro de cada fase. Logo, é necessário informar/qualificar quais são estes padrões e, buscar explicações sobre o porquê deles mudarem. Aqui reside a essência de uma abordagem processual.

- Ter uma fotografia do antes e depois do fenômeno (t0 e tn);

- Utilizar narrativas e mapas conceituais;

- Dividir a análise em fases;

- Identificar os eventos que marcam a troca de fase;

- Entender que a duração das fases pode variar;

- Explicar o padrão de cada fase;

- Explicar o "por que" de os padrões mudarem.

\section{COMO COLETAR DADOS EM ABORDAGENS} PROCESSUAIS?

Se a descrição do processo deve partir dos eventos, a alternativa que recomendamos para a coleta de dados é iniciar por apenas uma questão. Isso mesmo, uma pergunta que inicie com "conte-me o processo de xxxx". Este tipo de abordagem faz com que seu entrevistado seja capaz de contar todo o processo vivido enfatizando os eventos mais marcantes. As entrevistas intensivas são sugeridas por Charmaz (2009) como uma excelente fonte para examinar detalhadamente determinado tópico ou experiência.

É possível utilizar algumas estratégias alternativas como, por exemplo, levar uma folha A4 e pedir para que a pessoa desenhe o processo, divida em fases, aponte as relações entre os eventos. Esta estratégia pode ajudar muito na montagem do mapa conceitual e na narrativa. Tivemos alunos que foram mais longe e usaram Design Play, com Lego. Neste caso, solicitavam aos entrevistados que contassem a história do fenômeno e fossem inserindo os personagens de lego na bancada de trabalho... Ainda, pode-se usar da foto-elicitação (com fotografias do processo a ser analisado) para fazer com que os entrevistados possam recordar do processo vivenciado por eles.

Após as entrevistas, é importante fazer notas de campo. Elas serão fundamentais na escrita da narrativa. Use e abuse de dados secundários para suportar a narrativa.

Comece as entrevistas intensivas com UMA pergunta.

\section{QUAIS OS PASSOS NECESSÁRIOS PARA CONDUZIR UMA PESQUISA COM ABORDAGEM PROCESSUAL?}

Antes de apresentar uma proposta de passos, vale ressaltar que em pesquisas qualitativas e, especialmente, em pesquisa com abordagem processual a criatividade do pesquisador deve ser valorizada. Além disso, dada a quantidade de dados que um processo pode acumular, o pesquisador deve encontrar a forma que melhor se adapta à sua dinâmica. Dito isto, aqui vai a nossa proposta de etapas a seguir na realização de uma pesquisa desta natureza:

1) De tanto ter ouvido a história do fenômeno, certamente você será capaz de fazer um mapa prévio das fases e eventos principais. Faça para não perder os insights. 
2) O uso de um software de análise de dados qualitativos pode ajudar na organização da narrativa. Lembre-se que você pode ter mais de 30 versões da mesma história ao final de um processo de coleta de dados. Neste sentido, sugerimos que você categorize todos os eventos, colocando as datas nos nomes que atribuir aos mesmos. Depois é possível gerar relatórios do software por códigos e separar eles conforme as datas em que aconteceram! Vai facilitar muito seu trabalho!

3) Faça a narrativa por fases, isto é, toda vez você for citar um evento tem que colocar o que veio antes, o que causou o evento, o porquê do evento, porque eles tomam as decisões dentro de um contexto e o que gera para a continuidade do projeto.

4) Insira os dados secundários para garantir veracidade e triangulação.

5) Revise o mapa inicial, insira nele os eventos que destacou na narrativa e que passaram despercebidos na sua versão inicial.

6) Inicie o processo de análise dos dados pelo como. Analise o "como", ou seja, como é esta fase? Qual o padrão de comportamento que se apresenta ali? Tem atores prioritários? Julgamentos comuns? Escolhas? Quem sabe você até consegue dar um nome para esta fase e justificar o porquê.
7) Analise o "porquê", ou seja, por que os padrões de comportamento se alteram de uma fase para a outra? Há um padrão? Explique.

8) Envie seu mapa ou a sua narrativa aos entrevistados para que validem o resultado, afinal você construiu uma versão comum. Pode pedir um pequeno parecer sobre a nota dada.

\section{CONHECENDO OS PRINCIPAIS AUTORES E TEXTOS PARA O APROFUNDAMENTO NA ABORDAGEM PROCESSUAL}

São diversos os autores que trabalham utilizando a abordagem processual e que escrevem metodologicamente sobre seu rigor e passos fundamentais. Existe, inclusive, uma coletânea feita pela SAGE, intitulada "Handbook of Process Organization Studies", de 2017, que reúne os principais nomes e apresenta mais de 600 páginas dedicadas a fornecer um panorama do processo nos estudos organizacionais. Para este artigo, nós selecionamos os que são os nossos autores preferidos e alguns de seus textos, como forma de incentivá-los a dar o passo inicial nos estudos de processo (Tabela 1). É o mesmo material que apresentamos nas nossas aulas de metodologia qualitativa e que utilizamos como ponto de partida para familiarizar nossos orientandos.

Tabela 1

\begin{tabular}{|l|l|}
\hline \multicolumn{1}{|c|}{ Autor } & \multicolumn{1}{c|}{ Textos } \\
\hline Ann Langley & $\begin{array}{l}\text { Langley, A. (1999). Strategies for theorizing from process data. Academy of } \\
\text { Management review, 24(4), 691-710. } \\
\text { Langley, A., \& Abdallah, C. (2011). Templates and turns in qualitative studies } \\
\text { of strategy and management. In Building methodological bridges (pp. 201- } \\
\text { 235). Emerald Group Publishing Limited. }\end{array}$ \\
\hline Andrew H. Van de Vem & $\begin{array}{l}\text { Van de Ven, A. H., \& Poole, M. S. (1995). Explaining development and } \\
\text { change in organizations. Academy of management review, 20(3), 510-540. }\end{array}$ \\
\hline Lorenzo Bizzi & $\begin{array}{l}\text { Bizzi, L.; Langley, A. Studying processes in and around networks. Industrial } \\
\text { Marketing Management, v. 41, p. 224-234, 2012. }\end{array}$ \\
\hline
\end{tabular}

Fonte: elaborada pelas autoras

Por último, vale destacar que apesar de estarmos mais acostumados a nos deparar com pesquisas causais no mainstreaming dos estudos organizacionais, as pesquisas qualitativas tem ganhado cada vez mais espaço e a abordagem processual, em especial, tem apresentado grande potencial para os pesquisadores que olham para a gestão como algo dinâmico, em movimento. Entre as possibilidades de estudos, estão aqueles que se ocupam de rotinas organizacionais, estudos de cultura organizacional, processos institucionais, estratégia como prática, empreendedorismo como 
processo, inovação como processo, processos de conhecimento e aprendizagem organizacional, processos de liderança, entre tantos outros (LANGLEY; TSOUKAS, 2017).

Para aqueles que se questionam sobre a facilidade de publicar artigos que utilizam esta abordagem, nós ressaltamos que o rigor metodológico é fundamental.
Pesquisas processuais com rigor, que tenham originalidade e valor para o campo de pesquisa, são facilmente publicáveis. Temos tido experiências muito positivas nesse sentido, tanto em revistas brasileiras como em journals internacionais de grande impacto. Listamos na tabela 2 aqui somente alguns dos nossos estudos, para encorajar vocês a se aventurarem pelos caminhos do processo.

\section{Tabela 2}

\begin{tabular}{l} 
FACCIN, K.; WEGNER, D. ; BALESTRIN, A. (2020). How to orchestrate R\&D networks? The role of \\
orchestration subprocesses and collaborative practices over time. Creativity and Innovation \\
Management, v. 29, pp. 161-177. \\
\hline FACCIN, K.; BALESTRIN, A. ; VOLKMER MARTINS, B.; BITENCOURT, C. (2019). Knowledge-based \\
dynamic capabilities: a joint R\&D project in the French semiconductor industry. Journal of Knowledge \\
Management, v. 23, pp. 439-465. \\
\hline BEUTER JÚNIOR, N.; Faccin, K.; VOLKMER MARTINS, B.; BALESTRIN, A. (2019). Knowledge-Based \\
Dynamic Capabilities for Sustainable Innovation: The Case of the Green Plastic Project. Sustainability, \\
V. 11, pp. 2392. \\
\hline FACCIN, K.; BALESTRIN, A. (2018). The dynamics of collaborative practices for knowledge creation in \\
joint R\&D projects. Journal of Engineering and Technology Management, v. x, p. x. \\
\hline FACCIN, K.; BALESTRIN, A. ; BORTOLASO, I. (2016). The joint R\&D project: The case of the first Brazilian \\
microcontroller chip. RAUSP-E (SÃO PAULO), v. 51, pp. 87-102.
\end{tabular}

Fonte: elaborada pelas autoras

Por fim, entre os principais desafios que encontramos, principalmente no campo brasileiro de estudos organizacionais, está a necessidade de adoção de maior rigor metodológico na utilização da abordagem e, em torná-la mais conhecida, para que mais pesquisadores vejam suas possibilidades e a utilizem em seus estudos. Esperamos estar contribuindo para ultrapassar esses dois desafios com este artigo.

\section{REFERÊNCIAS}

BIZZI, L.; LANGLEY, A (2012). Studying processes in and around networks. Industrial Marketing Management, v. 41, pp. 224-234.

CHARMAZ, K. (2009). A construção da teoria fundamentada: guia prático para análise qualitativa. Porto Alegre: Artmed, 272 p.

LANGLEY, A. (1999). Strategies for theorizing from process data. Academy of Management review, v.24, n.4, pp. 691-710.
LANGLEY, A.; ABDALLAH, C. (2011). Templates and turns in qualitative studies of strategy and management. In: Building Methodological Bridges, pp. 201-235.

LANGLEY, A.; SMALLMAN, C.; TSOUKAS, H.; VAN DE VEN, A. H. (2013). Process Studies of Change in Organization and Management: Unveiling Temporality, Activity, and Flow. The Academy of Management Journal, v. 56, n. 1, pp. 1-13.

LANGLEY, A.; TRUAX, J.A. (1994). A Process Study of New Technology Adoption in Smaller Manufacturing Firms. Journal of Management Studies, v.31, pp. 619-652.

LANGLEY, A.; TSOUKAS, H.(ed).(2017).The SAGE Handbook of Process Organization Studies. SAGE Publications Ltda.

MOHR, L. B. (1982).Explaining Organizational Behavior. San Francisco: Jossey-Bass. 
VAN DE VEN, A. H. (1992). Suggestions for Studying Strategy Process. Strategic Management Journal, v.13, pp. 169-188.
VAN DE VEN, A. H.; Poole, M. S. (1995). Explaining development and change in organizations. Academy of Management Review, v.20, n.3, pp. 510-540.

\section{Como citar este artigo:}

Faccin, K., \& Volkmer Martins, B. (2022). Abordagem Processual em Pesquisa Qualitativa. Internext, 17(1), 128-134. https://doi.org/10.18568/internext.v17i1.685 